\title{
Methodology for the improvement of the AINA Code wall-model applied to DEMO WCPB blanket
}

\author{
M. Fabbri*, A. de Blas, A. Riego, J. Dies, I. Zamora, E. Baeza \\ Fusion Energy Engineering Laboratory (FEEL)-BarcelonaTech, Av. Diagonal 647, Pav. C, 08028 Barcelona, Spain
}

\section{H I G H L I G H T S}

- A new AINA code development methodology has been described and standardized.

- The 1D non-conservative responses have been compensated by scaling functions.

- The scaling functions have been determined covering different conditions.

\section{A R T I C L E I N F O}

\section{Article history:}

Received 20 September 2016

Received in revised form 28 April 2017

Accepted 5 May 2017

Available online $\mathrm{xxx}$

\section{Keywords:}

Safety

MCNP

AINA

WCPB

CFD

DEMO

Nuclear fusion

\begin{abstract}
A B S T R A C T
The present work describes and supports the methodology for the improvement of the wall model developed in AINA and its specific application to the Japanese DEMO Water Cooled Pebbled Bed. The set-up and application of this approach aims to obtain robust models by estimating the behavior of the studied systems as accurately as possible. These systems are represented in a simplified way. This requires the computation of a 3D radiation transport which has been carried out by means of MCNP6.1, ADVANTG and thermal-hydraulic calculations using ANSYS ${ }^{\circledR}$ Fluent $^{\circledR}$. Several CFD mesh typologies and discretizations have also been employed to test the Richardson theorem. In addition, 1D simplified models have also been created and optimized for their usage in AINA code. The temperature distribution also shows good agreement (within 7\%). In some cases the simplified models have not behaved in a conservative manner compared with the outcomes obtained for the 3D models. This observed absence of conservatism is intrinsic to the 1D approach. To cope with these effects, scaling functions have been determined as a ratio between the most conservative radial temperature distribution - computed by fully detailed 3D CFD and the 1D simplified model. The scaling functions will be applied to the AINA computed wall temperature distribution. To conclude, the determination and coherence of the result obtained using independent tools and approaches, ANSYS ${ }^{\circledR}$ Fluent ${ }^{\circledR}$ vs AINA thermal-hydraulic routines, lead us to recommend the proposed methodology.
\end{abstract}

(C) 2017 Elsevier B.V. All rights reserved.

\section{Introduction}

AINA, an acronym for Analyses of IN-vessel Accidents, is a safety code for fusion Tokamak reactors which estimates plasma-wall transients. Examples include ex-vessel Loss of Coolant Accidents (LOCA), over-fueling, and Loss of Plasma Control (LOPC). During the last ten years, several safety studies of plasma-wall transients have been undertaken for ITER [1-3] and for the Japanese DEMO design - Water Cooled Pebbled Bed (WCPB) [4] by means of specific AINA code versions. Moreover, in the coming years, further AINA versions of the European DEMO design (e.g. Helium Cooled Peb- bled Bed) are going to be developed within the Eurofusion frame. They will be identified as part of the European reference software in order to carry out the DEMO safety analyses [5]. For this purpose, an enhanced methodology for the creation and development of the AINA Code wall-model has been defined and described using the DEMO Water Cooled Pebbled Bed blanket, [6]. The set-up and application of this standard methodology aim to obtain robust models, estimating as accurately as possible the behavior of the studied systems. These systems are represented in a simplified way. In addition, the short AINA running time (a question of minutes) allows us to perform multiple simulations and establish boundaries for the worst scenarios - thus supporting the safety analysis.

\footnotetext{
* Corresponding author.

E-mail address: marco.fabbri@upc.edu (M. Fabbri).
} 


\section{Methodology}

The development of a new AINA code version for a specific blanket typology will require the modification of the core sections. (1) The plasma model has to be adjusted to take into account the different plasma parameters of the Tokamak (e.g. fusion power, elongation, triangularity and the dimensions of the Tokamak) from the previous configuration. Furthermore, a parameter cross-check by means of PROCESS [7] software will be carried out to check the validity of the changes introduced, as was done during previous versions of AINA [8]. (2) The introduction of new plasma facing components and new materials requires the adjustment of the impurity generation models. The presence of impurities affects the generation of bremsstrahlung and line radiation, cooling down the plasma. (3)The generation of a simplified new plasma-wall model is the most time-consuming phase and it should be generated according to the following steps. Firstly, fully detailed thermal-hydraulic and neutronics calculations have to be carried out to understand the behavior and the peculiarities of the system. Starting from these results, simplified models are drafted and compared using the same boundary conditions (BC) as the fully detailed calculations. Several iterations are needed to achieve the optimized configurations. At this stage, due to the simplifications introduced, the AINA wall model might not represent a conservative behavior compared with the fully detailed calculations. To cope with this, scaling functions (SF) are derived for different operation conditions and applied to the AINA thermal-wall results. The described methodology has been applied to WCPB and the main achievements are reported below. Please refer to [4] for point No. (1) and No. (2).

\section{Neutronics analysis}

Three-dimensional radiation transport calculations have been performed employing the MCNP6.1 (Monte Carlo N-Particle Transport Code) [9] with special attention to the neutronics performance of the WCPB blanket, focusing on the nuclear heating density (NHD), the tritium production and the neutron and gamma flux field. Coupled neutron/gamma transport calculations have been carried out using the reference cross-section library for the ITER Project, FENDL3.1b@300K (Evaluated Nuclear Data Library for Fusion Applications) [10], at room-temperature densities. A MCNP fully detailed blanket stand-alone model has been created using state-of-the-art methodology. SpaceClaim [11] and CATIA [12] software have been employed for the geometry defeaturing to allow the generation of MCNP geometry by means of SuperMC [13], limiting the volume differences due to simplification to less than $1 \%$. Volumetric Deuterium-Tritium Gaussian fusion source has been assumed and the neutron wall loading (NWL) normalized to $1 \mathrm{MW} / \mathrm{m}^{2}$. Reflective boundary conditions have been imposed on the blanket lateral sides for conservatism. The energy-dependent weight window variance reduction technique, generated by ADVANTG deterministic code [14], has been employed to obtain the quantities of interest with the desired statistical uncertainty and optimizing the computational effort. Results have been obtained and evaluated by means of tally and mesh-tally features. In addition, two 1D blanket simplified MNCP models have been generated in order to further optimize and reduce the computational effort enabling multiple future assessments. Starting from the 3D WCPB representation, forty-seven radial parallel layers have been created to preserve the in-vessel features as far as possible. In the end, they differ only in the material composition of the slices. Indeed, whereas one model employs the most representative material of the segmentation, the other uses a homogenized material mixture corresponding to the effective

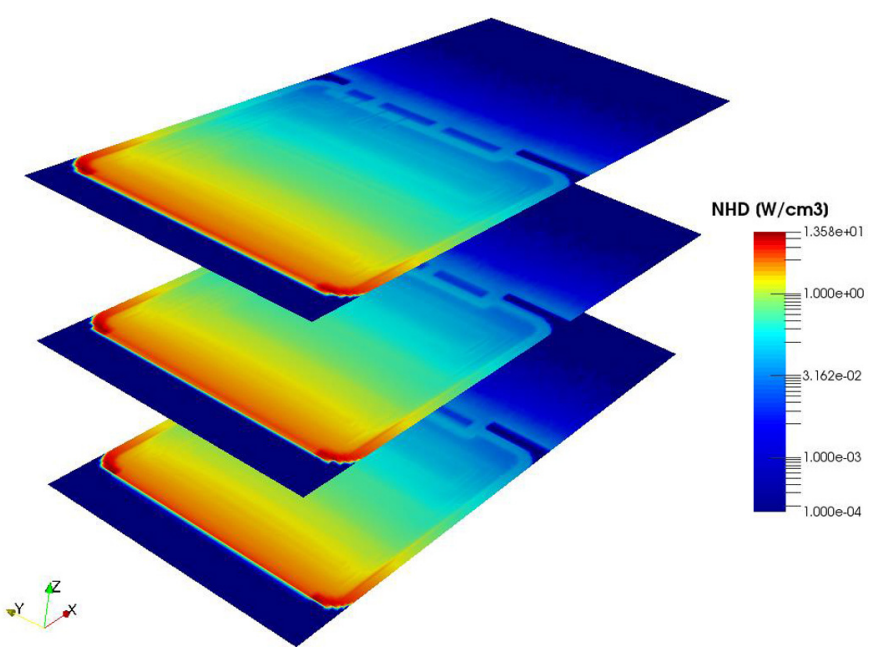

Fig. 1. Detailed radiation transport calculations: total nuclear heating distribution along several $Z$ planes.

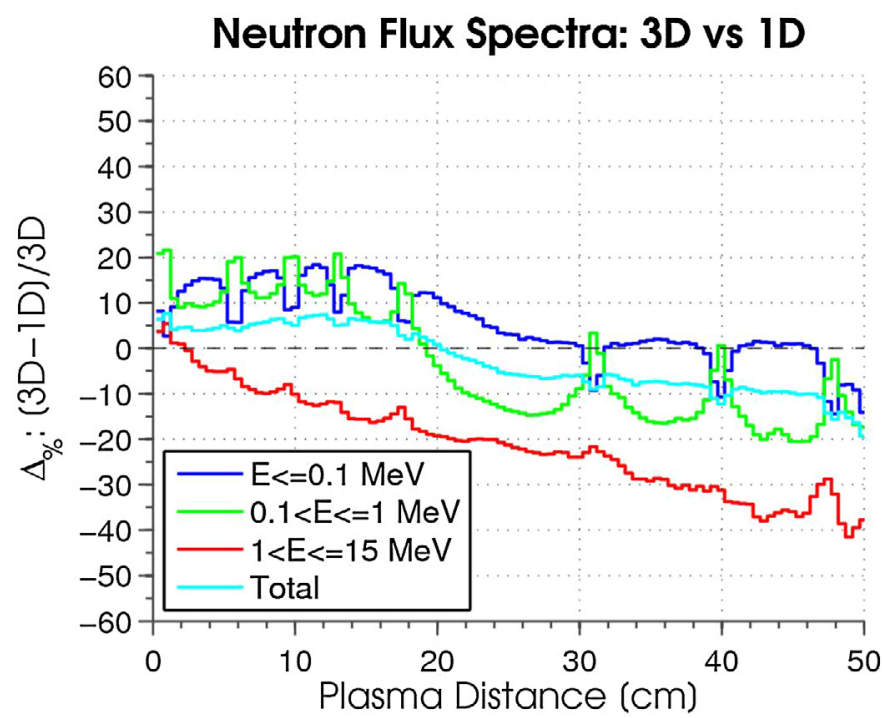

Fig. 2. Detailed radiation transport calculations: neutron flux spectra comparison.

cell composition. They are therefore identified as heterogeneous (1D-HET) and homogeneous (1D-HOM) models.

After a meaningful comparison, the first model has been preferred due to the greater integrated power deposition as well as the preservation of the NHD peaking profile. A selection of the radiation transport results is reported in Figs. 1 and 2. The NHD is dominated by neutrons in all of the analyzed cases. The neutron flux spectra distribution obtained from the 3D simulations is the most conservative along the radial direction. Considering the several differences between the fully detailed and simplified models, the results show good agreement. Indeed, both, the total neutron flux and the tritium production ratio (TPR) reach a maximum difference of $10 \%$. The main local discrepancies are located in the cooling areas due to the differences between the 3D and 1D models (see Fig. 3) and the back-scattering phenomena from surrounding areas which are intrinsically absent in the 1D approach. Furthermore, a smaller shielding performance of the 1D model breeding areas is observed triggering higher and more conservative temperatures on the thermal-hydraulic calculation of about $+5 \%$. Consequently, the 1D-HET nuclear responses have been used as input for the CFD calculation to further increase the safety margins. 

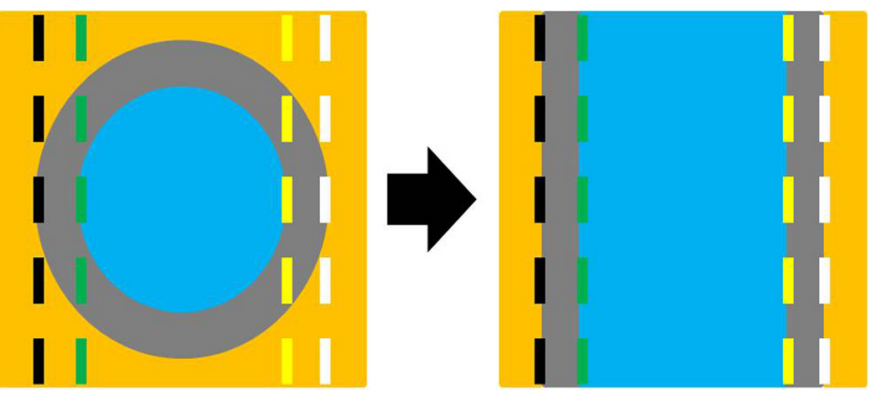

Fig. 3. Approximation of coolant regions in AINA wall model.

\section{Thermal-hydraulic analysis}

Thermal-hydraulic analyses have been computed by means of the computational fluid dynamics (CFD) ANSYS ${ }^{\circledR}$ Fluent $^{\circledR}$ v17.2 [15]. The original blanket geometrical model [16] has been simplified in order to remove non-relevant details for meshing proposal. Within this work, the use of automatic meshing has been discarded in favor of manual meshing in order to achieve a better control of mesh over small details such as the first wall (FW) channels and pipes. Therefore, Pointwise software [17] has been employed, resulting in a domain of $6.7 \mathrm{M}$ tetrahedra. The following $\mathrm{BC}$ have been applied to be conservative: adiabatic on $Y+\backslash Y$-, periodic boundary conditions on $Z+\backslash Z$ - thanks to the symmetrical geometry to reduce the domain size, Dirichlet BC on the Vacuum Vessel side $(T=598 \mathrm{~K})$ and Neumann BC on FW equal to $1.0 \mathrm{MW} / \mathrm{m}^{2}$. A constant heat transfer coefficient (HTC) and $T_{\text {bulk }}$ have been imposed on the coolant region according to [16], 13,285 W/ $/ \mathrm{m}^{2} / \mathrm{K}$ and $593 \mathrm{~K}$. The NHD computed by MNCP and the material temperature dependent properties have been introduced using in-house developed ANSYS User Defined Functions. The steady-state temperature field obtained is reported in Fig. 4. As expected, the temperature peaks have been found in the $X$ middle plane between the tubes, reaching a maximum of $1014 \mathrm{~K}$. No substantial gradients are observed in the $Z$ axis. More refined and polyhedral meshes have also been tested to comply with the Richardson theorem (as well as 2D very refined model): a difference of less than $5 \%$ has been found, confirming the independence of the mesh results. The most conservative temperature profile along the radial is determined and compared with the $1 \mathrm{D}$ solution, which is obtained using the AINA thermal-hydraulic routines based on a finite different scheme. It is worth remembering that the AINA blanket model employs the same radial discretization used in the 1D radiation transport calculations as well as the same BC $\backslash$ NHD parameters. Whereas an excellent agreement is obtained with a maximum difference of $7 \%$ close to the cooling channel, a consistent temperature underestimation is observed over the breeding area Fig. 5. This effect is mainly attributed to the 1D bigger cooling surface $\backslash$ volume ratio, the major distance between the cooling tubes apart from the axis plane and the one node coolant channel repre-

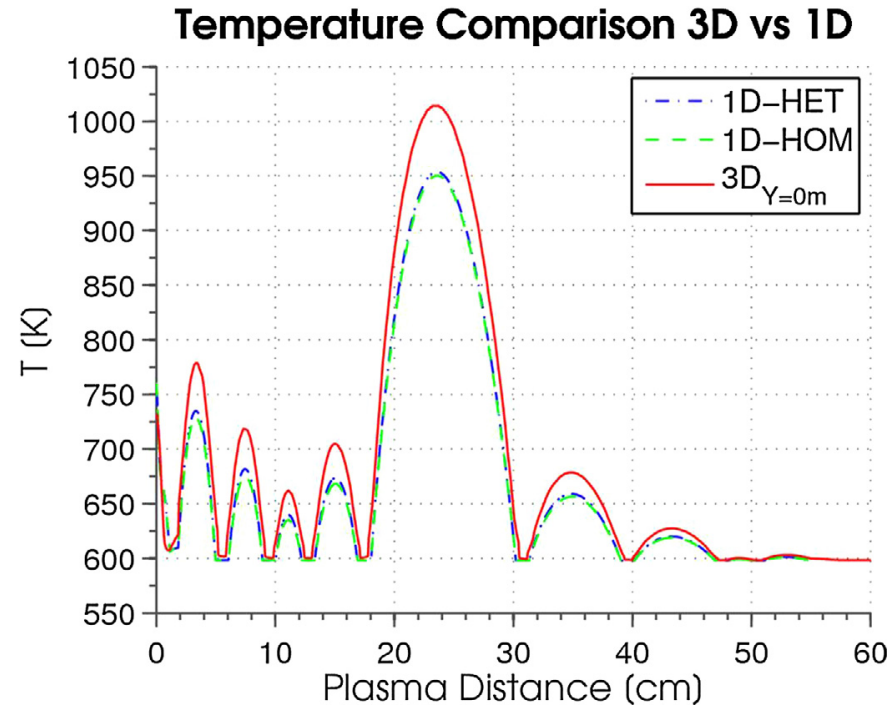

Fig. 5. 3D-1D temperature comparison@1 $1 \mathrm{MW} / \mathrm{m}^{2}$.

sentation Fig. 3. The non-conservatism observed is intrinsic to the 1D approach and thus unfortunately cannot be directly resolved. To cope with these effects, a scaling approach is proposed instead. The determination and coherence of the temperature distribution obtained using independent tools and approaches, ANSYS ${ }^{\circledR}$ Fluent ${ }^{\circledR}$ vs AINA thermal-hydraulic routines, justifies the proposed methodology, and hence validating the results.

\section{Scaling functions determination}

The use of simplified models instead of fully detailed ones could lead to an underestimated response and must be avoided. The innovative approach proposed in this paper is based on the application of scaling functions (SF) which compensate the AINA wall model responses (e.g. the temperature) only where the distribution results are less conservative than those of the fully detailed one. By doing this, an equal or more conservative response is obtained, thus enhancing the safety margins. For this purpose, several SF have been computed, covering all different conditions simulated by the AINA code. The LOPC and the LOCA functions are determined as a ratio between the most conservative radial temperature distribution computed by fully detailed 3D CFD and the 1D simplified model at steady-state condition using the same BC. As an example, Fig. 6 reports the LOPC SF obtained, which are a linear function of the NWL, with the exception of those from the breeding area around $25 \mathrm{~cm}$, where the maximum thermal conductivity value is reached. It should be noted that in case of intermediate conditions the SF are linearly interpolated.
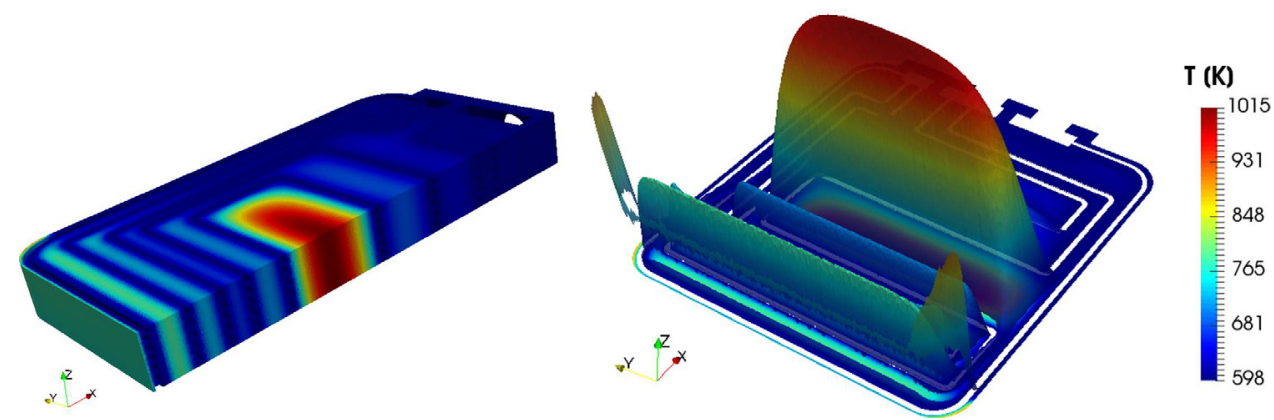

Fig. 4. WCPB temperature distribution-3D section vs 2D slice along $Z$ plane. 


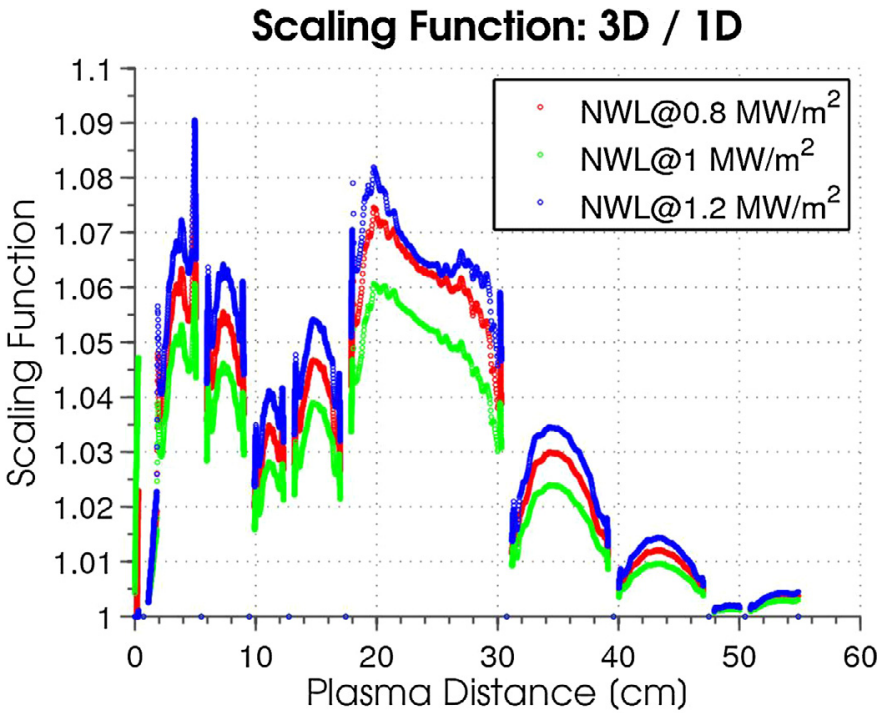

Fig. 6. Scaling functions for different neutron wall loadings.

\section{Conclusions}

The methodology for the development of a new AINA code version has been described and its application to the Japanese DEMO WCPB focusing on the wall-model generation reported. Threedimensional radiation transport calculations by means of MCNP6.1 code and the state-of-art methodology have been carried out and compared with the 1D simplified models, which are valuable for future sensibility assessments. The NHD and the neutron/photon flux show good agreement, within 10\%: the major differences are located in the cooling areas due to different approaches and the absence of backscattering phenomena from the surroundings areas. Furthermore, TPR have been computed and are available for future applications. Using the computed NHD, the WCPB 3D thermal hydraulic simulations have been carried out and their results exposed. As expected, the temperature peaks are located between the cooling tubes. Mesh sensibility studies fulfilling the Richardson theorem have been carried out, obtaining differences of less than $5 \%$, thus proving the mesh response independence. Thanks to non substantial gradients along the $Z$ direction, a 1D simplified model has been iteratively built and adjusted, achieving a good agreement with the fully detailed simulation and yielding a maximum absolute temperature difference of about $7 \%$. The determination and coherence of the temperature distribution obtained using independent tools and approaches, ANSYS ${ }^{\circledR}$ Fluent ${ }^{\circledR}$ vs AINA thermal-hydraulic routines, supports the proposed methodology, hence validating the results. Nevertheless, the $1 \mathrm{D}$ non-conservative temperature field, where present, is compensated by the application of scaling functions, obtaining a perfect match with the most conservative 3D distribution. The scaling functions correspond to the ratios between the most conservative radial distribution in the fully detailed and the $1 \mathrm{D}$ simulations and they have been computed to cover the whole operational areas including accidental scenarios. For intermediate conditions, the SF are linearly interpolated. Thanks to this robust methodology, starting from fully detailed neutronics and thermal-hydraulic results, a simplified and conservative wall model will be implemented in AINA from now on, obtaining reliable results in a short calculation time. It is worth remembering that the formulation of simplified models without the full and rigorous comparison with the fully detailed model could result in non-conservative results, which are avoided using this methodology. Future developments will be focused on the impact of the variation of NHD (due to temperatures, different cross sections, Doppler effect broadening) on the thermal-wall field as well as the determination of the effect of this methodology on the AINA safety analysis.

\section{Acknowledgements}

The authors thank the International Fusion Energy Research Centre (IFERC) for providing all necessary information to perform this development. This work was partly supported by the Broader Approach Activities. The views and opinions expressed herein do not necessarily reflect those of IFERC and Fusion of Energy.

\section{References}

[1] J. Rivas, J. Dies, ITER safety studies: the effect of two simultaneous perturbations during a loss of plasma control transient, Proceedings of the 11 th International Symposium on Fusion Nuclear Technology-11 (ISFNT-11) Barcelona, Spain, 15-20 September, 2013, Fusion Eng. Des. 89 (2014) 2043-2047, http://dx.doi.org/10.1016/j.fusengdes.2014.04.010.

[2] J. Dies, M. Dapena, M. Ramon, R. Lopez, J. García, J.C. Rivas, A. Calvo, S. Reyes, AINA safety code, a review of loss of plasma control transients in ITER: sudden increase in fuelling rate, sudden increase of auxiliary heating, Fusion Sci. Technol. 56 (1) (2009) 31-37, http://dx.doi.org/10.13182/FST09-A8871.

[3] J. Rivas, J. Dies, Safety studies: review of loss of plasma control transients in ITER with AINA 3.0 code, Fusion Eng. Des. 88 (9-10) (2013) 2709-2713, http://dx.doi.org/10.1016/j.fusengdes.2013.02.134.

[4] J. Rivas, M. Nakamura, Y. Someya, K. Hoshino, N. Asakura, H. Takase, Y. Miyoshi, H. Utoh, K. Tobita, J. Dies, A. de Blas, A. Riego, M. Fabbri, Safety studies of plasma-wall events with $\{$ AINA $\}$ code for Japanese $\{$ DEMO $\}$, Proceedings of the 12th International Symposium on Fusion Nuclear Technology-12 (ISFNT-12), Fusion Eng. Des. 109-111 (Part B) (2016) 1653-1657, http://dx.doi.org/10.1016/j.fusengdes.2015.10.037.

[5] M.T. Porfiri, Executive Summary of the EFDA Tasks: Wp13-sys-04 efda_d 2 hf6n2 v.1.0, Tech. rep., ENEA, 2014.

[6] M. Enoeda, H. Tanigawa, T. Hirose, M. Nakajima, S. Sato, K. Ochiai, C. Konno, Y. Kawamura, T. Hayashi, T. Yamanishi, T. Hoshino, M. Nakamichi, H. Tanigawa, H. Nishi, S. Suzuki, K. Ezato, Y. Seki, K. Yokoyama, R\&D status on water cooled ceramic breeder blanket technology, Proceedings of the 11th International Symposium on Fusion Nuclear Technology-11 (ISFNT-11) Barcelona, Spain, 15-20 September, 2013, Fusion Eng. Des. 89 (7-8) (2014) 1131-1136, http:// dx.doi.org/10.1016/j.fusengdes.2014.01.035.

[7] M. Kovari, R. Kemp, H. Lux, P. Knight, J. Morris, D. Ward, Process: a systems code for fusion power plants - Part 1: Physics, Fusion Eng. Des. 89 (12) (2014) 3054-3069, http://dx.doi.org/10.1016/j.fusengdes.2014.09.018.

[8] J. Dies, Progress in AINA Development for Demo, efda_d_2lm82t v.1.0, Tech. rep., UPC-FEEL, 2015.

[9] X.-M.C. Team, MCNP A General Monte Carlo N-Particle Transport Code, Version 6, LANL, April 2014.

[10] R.C.N.D. Lopez Aldama, Fendl-3.1b: Processing the Evaluated Nuclear Data Library for Fusion Applications, Tech. rep., International Atomic Energy Agency, 2016, URL https://www-nds.iaea.org/publications/indc/indc-nds0611/.

[11] ANSYS, ANSYS SpaceClaim 2016, 2016, URL http://www.spaceclaim.com.

[12] Dass, CATIA V5, 2017.

[13] Y. Wu, J. Song, H. Zheng, G. Sun, L. Hao, P. Long, L. Hu, CAD-based Monte Carlo program for integrated simulation of nuclear system SuperMC, Joint International Conference on Supercomputing in Nuclear Applications and Monte Carlo 2013, $\{$ SNA $\}+\{$ MC $\}$ 2013. Pluri- and Trans-disciplinarity, Towards New Modeling and Numerical Simulation Paradigms, Ann. Nucl. Energy 82 (2015) 161-168, http://dx.doi.org/10.1016/j.anucene.2014.08.058.

[14] J.C. Wagner, et al., Review of hybrid (deterministic/Monte Carlo) radiation transport methods, codes, and applications at oak ridge national laboratory, Prog. Nucl. Sci. Technol. 2 (2011) 808-814.

[15] ANSYS, ANSYS Fluent (C) User Manual v17. 2, 2015, URL http://www.ansys. $\mathrm{com} /$.

[16] J.C. Ribas, Private E-mail Communication, Tech. rep., UPC-IFERC, 2015

[17] POINTWISE, 2017. URL http://www.pointwise.com/pointwise/. 